\title{
Direct and Inverse Problems for Sturm-Liouville Operator Which Has Discontinuity Conditions and Coulomb Potential
}

\author{
Yalçın Güldü and Merve Arslantaş \\ Department of Mathematics, Faculty of Sciences, Cumhuriyet University, 58140 Sivas, Turkey \\ Correspondence should be addressed to Yalçın Güldü; yguldu@gmail.com
}

Received 28 November 2013; Accepted 16 December 2013; Published 18 February 2014

Academic Editors: L. Denis and W. Klingenberg

Copyright ( 2014 Y. Güldü and M. Arslantaş. This is an open access article distributed under the Creative Commons Attribution License, which permits unrestricted use, distribution, and reproduction in any medium, provided the original work is properly cited.

We give a derivation of the main equation for Sturm-Liouville operator with Coulomb potential and prove its unique solvability. Using the solution of the main equation, we get an algorithm for the solution of the inverse problem.

\section{Introduction}

In this study, we consider the boundary value problem $L$ :

$$
\begin{gathered}
l y=-y^{\prime \prime}+\left(\frac{C}{x}+q(x)\right) y=\lambda y, \quad \lambda=\rho^{2}, 0<x<\pi, \\
U(y):=y(0)=0, \quad V(y):=y(\pi)=0, \\
y(d+0)=\alpha y(d-0), \\
y^{\prime}(d+0)=\alpha^{-1} y^{\prime}(d-0),
\end{gathered}
$$

where $\lambda=\rho^{2}$ is a spectral parameter, $C>0, \alpha \in \mathbb{R}^{+} \backslash\{1\}, d \in$ $(\pi / 2, \pi), q(x)$ is a real valued bounded function, and $q(x) \in$ $L_{2}(0, \pi)$.

In spectral theory, the inverse problem is the usual name for any problem in which it is required to ascertain the spectral data that will determine a differential operator uniquely and a method of construction of this operator from the data. This kind of problem was first formulated and investigated by Ambarzumian in 1929 [1]. Since 1946, various forms of the inverse problem have been considered by numerous authors, Borg [2], Levinson [3], Levitan [4], and so forth, and now there exists extensive literature in [5-9]. Later, the inverse problems having specified singularities were considered by a number of authors [10-12].
The method of spectral mappings is an impressive device for investigating a profound class of inverse problems not only for Sturm-Liouville operators, but also for other more complicated classes of operators such as differential operators of arbitrary orders, differential operators with singularities, and pencils of operators. We apply the method of spectral mappings to the solution of the inverse problem for the Sturm-Liouville operator on a finite interval.

In the method of spectral mappings we begin from Cauchy's integral formula for analytic functions. We apply this theorem in the complex plane of the spectral parameter for specially constructed analytic functions having Coulomb singularity connected with the given spectral characteristics. This permits us to reduce the inverse problem to the so-called main equation which is a linear equation in a corresponding Banach space of sequences.

In this paper, first, integral representation for solution which satisfies certain initial conditions of differential equation generated by Sturm-Liouville operator with Coulomb potential is handled. Second, properties of spectral characteristics and uniqueness theorems for solution of inverse problem are discussed. After that we give a derivation of the main equation and prove its unique solvability. Finally, we obtain an algorithm for the solution of the inverse problem by using the solution of the main equation. 


\section{Preliminaries}

We define

$$
(\Gamma y)(x)=y^{\prime}-u(x) y \quad \text { where } u(x)=C \ln x \in L_{2}(0, \pi),
$$

and let us write the expression of the left-hand side of (1) as follows:

$$
l(y)=-[(\Gamma y)(x)]^{\prime}-u(x)(\Gamma y)(x)-u^{2}(x) y+q(x) y .
$$

Here, $(\Gamma y)(x)$ has a limit as $x \rightarrow 0^{+}$; that is, $\lim _{x \rightarrow 0}(\Gamma y)(x)=(\Gamma y)(0)$.

Theorem 1 (see [13]). Solution of the problem $L$ which satisfies the initial conditions $\left(\begin{array}{l}y_{1} \\ y_{2}\end{array}\right)(0)=\left(\begin{array}{c}1 \\ i \rho\end{array}\right)$ and the discontinuity conditions (3) has the following form.

If $x<d$,

$$
\begin{aligned}
y_{1}= & e^{i \rho x}+\int_{-x}^{x} K_{11}(x, t) e^{i \rho t} d t, \\
y_{2}= & i \rho e^{i \rho x}+b(x) e^{i \rho x} \\
& +\int_{-x}^{x} K_{21}(x, t) e^{i \rho t} d t+i \rho \int_{-x}^{x} K_{22}(x, t) e^{i \rho t} d t,
\end{aligned}
$$

if $x>d$,

$$
\begin{aligned}
y_{1}= & \alpha^{+} e^{i \rho x}+\alpha^{-} e^{i \rho(2 d-x)}+\int_{-x}^{x} K_{11}(x, t) e^{i \rho t} d t, \\
y_{2}= & i \rho\left(\alpha^{+} e^{i \rho x}-\alpha^{-} e^{i \rho(2 d-x)}\right) \\
& +b(x)\left[\alpha^{+} e^{i \rho x}+\alpha^{-} e^{i \rho(2 d-x)}\right] \\
& +\int_{-x}^{x} K_{21}(x, t) e^{i \rho t} d t+i \rho \int_{-x}^{x} K_{22}(x, t) e^{i \rho t} d t,
\end{aligned}
$$

where

$$
\begin{gathered}
b(x)=-\frac{1}{2} \int_{0}^{x}\left[u^{2}(s)-q(s)\right] e^{-(1 / 2) \int_{s}^{x} u(t) d t} d s \\
K_{11}(x, x)=\frac{\alpha^{+}}{2} u(x), \quad K_{11}(x,-x)=0, \\
K_{11}(x, 2 d-x+0)-K_{11}(x, 2 d-x-0)=\frac{\alpha^{-}}{2} u(x) \\
K_{21}(x, x)=b^{\prime}(x)-\frac{1}{2} \int_{0}^{x}\left[u^{2}(s)-q(s)\right] K_{11}(s, s) d s \\
\quad-\frac{1}{2} \int_{0}^{x} u(s) K_{21}(s, s) d s, \\
K_{22}(x, x)=-\frac{\alpha^{+}}{2}[u(x)+2 b(x)] \\
\frac{\partial K_{i j}(x, \cdot)}{\partial x}, \frac{\partial K_{i j}(x, \cdot)}{\partial t} \in L_{2}(0, \pi), \quad i, j=1,2 .
\end{gathered}
$$

In this part, we consider the properties of the spectrum of the problem $L$. In the case of $C=0$ and $q(x) \equiv 0$, the problem $L$ is denoted by $L_{0}$. Let us verify that a solution $\varphi(x, \lambda)$ of $(1)$ with the initial conditions $\varphi(0, \lambda)=0$ and $(\Gamma \varphi)(0, \lambda)=\sqrt{\lambda}=\rho$ and discontinuity conditions (3). When $C=0$ and $q(x) \equiv 0$, it is easily shown that solution $\varphi_{0}(x, \lambda)$ with the same conditions has the form

$$
\begin{aligned}
& \varphi_{0}(x, \lambda)= \begin{cases}\frac{1}{\sqrt{\lambda}} \sin \sqrt{\lambda} x, & x<d, \\
\frac{\alpha^{+}}{\sqrt{\lambda}} \sin \sqrt{\lambda} x+\frac{\alpha^{-}}{\sqrt{\lambda}} \sin \sqrt{\lambda}(2 d-x), & x>d,\end{cases} \\
& \left(\Gamma \varphi_{0}\right)(x, \lambda)= \begin{cases}\cos \sqrt{\lambda} x, & x<d, \\
\alpha^{+} \cos \sqrt{\lambda} x-\alpha^{-} \cos \sqrt{\lambda}(2 d-x), & x>d .\end{cases}
\end{aligned}
$$
$\Delta_{0}(\lambda)$ :

We denote the characteristic function of the problem $L_{0}$ by

$$
\Delta_{0}(\lambda)=\varphi_{0}(\pi, \lambda)=\frac{\alpha^{+}}{\sqrt{\lambda}} \sin \sqrt{\lambda} \pi+\frac{\alpha^{-}}{\sqrt{\lambda}} \sin \sqrt{\lambda}(2 d-\pi) .
$$

The roots $\lambda_{n}^{0}$ of equation $\Delta_{0}(\lambda)=0$ for $n \in \mathbb{N}$ are the eigenvalues of the problem $L_{0}$.

On the other hand, normalizing constants $\alpha_{n}$ of the problem L are defined as follows:

$$
\alpha_{n}:=\int_{0}^{\pi} \varphi^{2}\left(x, \lambda_{n}\right) d x
$$

Lemma 2 (see [13]). The eigenvalues of the problem L satisfy the asymptotic equality

$$
\sqrt{\lambda_{n}}=\rho_{n}=\rho_{n}^{0}+\frac{d_{n}}{\left(\rho_{n}^{0}\right)^{2}}+\frac{\delta_{n}}{\left(\rho_{n}^{0}\right)^{2}}
$$

where $\delta_{n} \in \ell_{2}, \rho_{n}^{0}=n+h_{n}$, sup $\left|h_{n}\right| \leq M, d_{n} \in \ell_{\infty}$, and

$$
d_{n}=\frac{\alpha^{+} \cos \sqrt{\lambda_{n}^{0}} \pi+\alpha^{-} \cos \sqrt{\lambda_{n}^{0}}(2 d-\pi)}{2 \dot{\Delta}_{0}\left(\lambda_{n}^{0}\right)} u(\pi) .
$$

Lemma 3 (see [13]). The normalizing constants of the problem $L$ satisfy the asymptotic equality

$$
\alpha_{n}=\alpha_{n}^{0}+\kappa_{n}
$$

where $\kappa_{n} \in \ell_{2}$.

On the other hand, eigenfunctions $\varphi\left(x, \lambda_{n}\right)$ of problem $L$ have the following asymptotic behaviour.

For $x<d$,

$$
\begin{aligned}
\varphi\left(x, \lambda_{n}\right)= & \frac{1}{\sqrt{\lambda_{n}}} \sin \sqrt{\lambda_{n}} x \\
& +\frac{1}{\sqrt{\lambda_{n}}} \int_{0}^{x} \widetilde{K}_{11}(x, t) \sin \sqrt{\lambda_{n}} t d t
\end{aligned}
$$




$$
\begin{aligned}
& =\frac{1}{\sqrt{\lambda_{n}}} \sin \sqrt{\lambda_{n}} x+O\left(\frac{e^{\left|\operatorname{Im} \sqrt{\lambda_{n}}\right| x}}{\sqrt{\lambda_{n}}}\right) \\
& =O\left(\frac{e^{\left|\operatorname{Im} \sqrt{\lambda_{n}}\right| x}}{\sqrt{\lambda_{n}}}\right), \quad \lambda_{n} \longrightarrow \infty, \\
(\Gamma \varphi)\left(x, \lambda_{n}\right) & =\cos \sqrt{\lambda_{n}} x+O\left(\frac{e^{\left|\operatorname{Im} \sqrt{\lambda_{n}}\right| x}}{\sqrt{\lambda_{n}}}\right) \\
& =O\left(e^{\left|\operatorname{Im} \sqrt{\lambda_{n}}\right| x}\right), \quad \lambda_{n} \longrightarrow \infty .
\end{aligned}
$$

For $x>d$,

$$
\begin{array}{r}
\varphi\left(x, \lambda_{n}\right)=\frac{\alpha^{+}}{\sqrt{\lambda_{n}}} \sin \sqrt{\lambda_{n}} x+\frac{\alpha^{-}}{\sqrt{\lambda_{n}}} \sin \sqrt{\lambda_{n}}(2 d-x) \\
+O\left(\frac{e^{\left|\operatorname{Im} \sqrt{\lambda_{n}}\right| x}}{\sqrt{\lambda_{n}}}\right)=O\left(\frac{e^{\left|\operatorname{Im} \sqrt{\lambda_{n}}\right| x}}{\sqrt{\lambda_{n}}}\right), \\
(\Gamma \varphi)\left(x, \lambda_{n}\right)=\alpha^{+} \cos \sqrt{\lambda_{n}} x-\alpha^{-} \cos \sqrt{\lambda_{n}}(2 d-x) \\
+O\left(\frac{e^{\left|\operatorname{Im} \sqrt{\lambda_{n}}\right| x}}{\sqrt{\lambda_{n}}}\right)=O\left(e^{\left|\operatorname{Im} \sqrt{\lambda_{n}}\right| x}\right), \\
\lambda_{n} \longrightarrow \infty .
\end{array}
$$

We consider the inverse problem of the reconstruction of problem $L$ according to Weyl function. For this reason, we can also consider a boundary value problem $\widetilde{L}=L(\widetilde{q}(x))$ of the same form together with $L$.

Assume that $\Phi(x, \lambda)$ is a solution of (1) which satisfies the conditions $\Phi(0, \lambda)=1$ and the conditions (3).

Let $C(x, \lambda)$ and $\Psi(x, \lambda)$ denote solutions of (1) that satisfy the initial conditions $\left(\begin{array}{c}C \\ C^{\prime}\end{array}\right)(0, \lambda)=\left(\begin{array}{l}1 \\ 0\end{array}\right)$ and $\left(\begin{array}{l}\Psi \\ \Psi^{\prime}\end{array}\right)(\pi, \lambda)=$ $\left(\begin{array}{c}0 \\ -1\end{array}\right)$, respectively, and jump conditions (3).

We set $M(\lambda):=\Phi^{\prime}(0, \lambda)$. The functions $\Phi(x, \lambda)$ and $M(\lambda)$ are called the Weyl solution and Weyl function of the boundary value problem $L$, respectively.

It is obvious that

$$
\begin{gathered}
\Phi(x, \lambda)=\frac{\Psi(x, \lambda)}{\Delta(\lambda)}=C(x, \lambda)+M(\lambda) \varphi(x, \lambda), \\
M(\lambda)=\frac{\Psi^{\prime}(0, \lambda)}{\Delta(\lambda)} \\
\langle\Phi(x, \lambda), \varphi(x, \lambda)\rangle \equiv 1 .
\end{gathered}
$$

Therefore, the Weyl function is meromorphic with simple poles in the points $\lambda=\lambda_{n}, n \geq 0$.

Lemma 4. If $M(\lambda)=\widetilde{M}(\lambda)$, then $L=\widetilde{L}$. Therefore, the boundary value problem $L$ is uniquely determined by the Weyl function.
Proof. We define the matrix $P(x, \lambda)=\left[P_{j k}(x, \lambda)\right]_{j, k=1,2}$ by the formula

$$
P(x, \lambda)\left(\begin{array}{cc}
\widetilde{\varphi} & \widetilde{\Phi} \\
\widetilde{\varphi}^{\prime} & \widetilde{\Phi}^{\prime}
\end{array}\right)=\left(\begin{array}{cc}
\varphi & \Phi \\
\varphi^{\prime} & \Phi^{\prime}
\end{array}\right)
$$

Using (19) and (20), we get

$$
\begin{gathered}
P_{11}(x, \lambda)=\Phi(x, \lambda) \widetilde{\varphi}^{\prime}(x, \lambda)-\varphi(x, \lambda) \widetilde{\Phi}^{\prime}(x, \lambda) \\
P_{12}(x, \lambda)=\varphi(x, \lambda) \widetilde{\Phi}(x, \lambda)-\Phi(x, \lambda) \widetilde{\varphi}(x, \lambda) \\
P_{21}(x, \lambda)=\Phi^{\prime}(x, \lambda) \widetilde{\varphi}^{\prime}(x, \lambda)-\varphi^{\prime}(x, \lambda) \widetilde{\Phi}^{\prime}(x, \lambda) \\
P_{22}(x, \lambda)=\varphi^{\prime}(x, \lambda) \widetilde{\Phi}(x, \lambda)-\Phi^{\prime}(x, \lambda) \widetilde{\varphi}(x, \lambda), \\
\varphi(x, \lambda)=P_{11}(x, \lambda) \widetilde{\varphi}(x, \lambda)+P_{12}(x, \lambda) \widetilde{\varphi}^{\prime}(x, \lambda) \\
\varphi^{\prime}(x, \lambda)=P_{21}(x, \lambda) \widetilde{\varphi}(x, \lambda)+P_{22}(x, \lambda) \widetilde{\varphi}^{\prime}(x, \lambda) \\
\Phi(x, \lambda)=P_{11}(x, \lambda) \widetilde{\Phi}(x, \lambda)+P_{12}(x, \lambda) \widetilde{\Phi}^{\prime}(x, \lambda) \\
\Phi^{\prime}(x, \lambda)=P_{21}(x, \lambda) \widetilde{\Phi}(x, \lambda)+P_{22}(x, \lambda) \widetilde{\Phi}^{\prime}(x, \lambda) .
\end{gathered}
$$

On the other hand, from the hypothesis $M(\lambda)=\widetilde{M}(\lambda)$, by using (17) and (21), we get

$$
\begin{gathered}
P_{11}(x, \lambda)=\widetilde{\varphi}^{\prime}(x, \lambda) C(x, \lambda)-\varphi(x, \lambda) \widetilde{C}^{\prime}(x, \lambda) \\
P_{12}(x, \lambda)=\varphi(x, \lambda) \widetilde{C}(x, \lambda)-C(x, \lambda) \widetilde{\varphi}(x, \lambda) \\
P_{21}(x, \lambda)=C^{\prime}(x, \lambda) \widetilde{\varphi}^{\prime}(x, \lambda)-\varphi^{\prime}(x, \lambda) \widetilde{C}(x, \lambda) \\
P_{22}(x, \lambda)=\varphi^{\prime}(x, \lambda) \widetilde{C}(x, \lambda)-C^{\prime}(x, \lambda) \widetilde{\varphi}(x, \lambda) .
\end{gathered}
$$
$\lambda$.

So, from (23), when $M(\lambda)=\widetilde{M}(\lambda), P_{j k}(x, \lambda)$ are entire on Since

$$
\langle\Psi(x, \lambda), \varphi(x, \lambda)\rangle=\Delta(\lambda),
$$

relations (17), (21), and (24) yield

$$
\begin{aligned}
P_{11}(x, \lambda)= & \widetilde{\varphi}^{\prime}(x, \lambda) \frac{\Psi(x, \lambda)}{\Delta(\lambda)}-\varphi(x, \lambda) \frac{\widetilde{\Psi}^{\prime}(x, \lambda)}{\widetilde{\Delta}(\lambda)} \\
= & 1+(\widetilde{\varphi}(x, \lambda)-\varphi(x, \lambda)) \frac{\widetilde{\Psi}^{\prime}(x, \lambda)}{\widetilde{\Delta}(\lambda)} \\
& -\left(\frac{\widetilde{\Psi}(x, \lambda)}{\widetilde{\Delta}(\lambda)}-\frac{\Psi(x, \lambda)}{\Delta(\lambda)}\right) \widetilde{\varphi}^{\prime}(x, \lambda),
\end{aligned}
$$




$$
\begin{aligned}
& P_{12}(x, \lambda)=\varphi(x, \lambda) \frac{\widetilde{\Psi}(x, \lambda)}{\widetilde{\Delta}(\lambda)}-\widetilde{\varphi}(x, \lambda) \frac{\Psi(x, \lambda)}{\Delta(\lambda)} \\
& =\left(\frac{\widetilde{\Psi}(x, \lambda)}{\widetilde{\Delta}(\lambda)}-\frac{\Psi(x, \lambda)}{\Delta(\lambda)}\right) \varphi(x, \lambda) \\
& -(\widetilde{\varphi}(x, \lambda)-\varphi(x, \lambda)) \frac{\Psi(x, \lambda)}{\Delta(\lambda)}, \\
& P_{21}(x, \lambda)=\widetilde{\varphi}^{\prime}(x, \lambda) \frac{\Psi^{\prime}(x, \lambda)}{\Delta(\lambda)}-\varphi^{\prime}(x, \lambda) \frac{\widetilde{\Psi}^{\prime}(x, \lambda)}{\widetilde{\Delta}(\lambda)} \\
& =\left(\frac{\widetilde{\varphi}^{\prime}(x, \lambda)}{\Delta(\lambda)}-\frac{\varphi^{\prime}(x, \lambda)}{\widetilde{\Delta}(\lambda)}\right) \widetilde{\Psi}^{\prime}(x, \lambda) \\
& -\left(\widetilde{\Psi}^{\prime}(x, \lambda)-\Psi^{\prime}(x, \lambda)\right) \frac{\widetilde{\varphi}^{\prime}(x, \lambda)}{\Delta(\lambda)}, \\
& P_{22}(x, \lambda)=\varphi^{\prime}(x, \lambda) \frac{\widetilde{\Psi}(x, \lambda)}{\widetilde{\Delta}(\lambda)}-\widetilde{\varphi}(x, \lambda) \frac{\Psi^{\prime}(x, \lambda)}{\Delta(\lambda)} \\
& =1+\varphi^{\prime}(x, \lambda)\left(\frac{\widetilde{\Psi}(x, \lambda)}{\widetilde{\Delta}(\lambda)}-\frac{\Psi(x, \lambda)}{\Delta(\lambda)}\right) \\
& -(\widetilde{\varphi}(x, \lambda)-\varphi(x, \lambda)) \frac{\Psi^{\prime}(x, \lambda)}{\Delta(\lambda)} .
\end{aligned}
$$

Let $G_{\delta}=\left\{\lambda: \lambda=\rho^{2},\left|\rho-\rho_{n}^{0}\right| \geq \delta, n=0, \pm 1, \pm 2, \ldots\right\}$ where $\delta$ is sufficiently small number. Since $|\Delta(\lambda)|>C_{\delta} e^{|\operatorname{Im\rho }| \pi}$ in $G_{\delta}$ and from the representations of the solutions

$$
\begin{gathered}
\varphi(x, \lambda)=\varphi_{0}(x, \lambda)+\int_{-x}^{x} K_{11}(x, t) \sin \rho t d t, \\
\Psi(x, \lambda)=\Psi_{0}(x, \lambda)+\int_{-(\pi-x)}^{\pi-x} N_{11}(x, t) \sin \rho t d t
\end{gathered}
$$

we see that $P_{j k}(x, \lambda)$ are bounded with respect to $\lambda$. Hence it is clear from Liouville's theorem that $P_{j k}(x, \lambda)$ do not depend on $\lambda$. Therefore, from the asymptotic formulae of the solutions $\varphi(x, \lambda), \Psi(x, \lambda)$,

$$
\begin{aligned}
& \lim _{\lambda \rightarrow \infty}(\widetilde{\varphi}(x, \lambda)-\varphi(x, \lambda)) \frac{\widetilde{\Psi}^{\prime}(x, \lambda)}{\widetilde{\Delta}(\lambda)}=0, \\
& \lim _{\lambda \rightarrow \infty}\left(\frac{\widetilde{\Psi}(x, \lambda)}{\widetilde{\Delta}(\lambda)}-\frac{\Psi(x, \lambda)}{\Delta(\lambda)}\right) \widetilde{\varphi}^{\prime}(x, \lambda)=0
\end{aligned}
$$

for all $x \in(0, d) \cup(d, \pi)$ and for $\lambda \in R$. Finally,

$$
\lim _{\lambda \rightarrow \infty}\left(P_{11}(x, \lambda)-1\right)=0
$$

uniformly with respect to $x$. Therefore,

$$
\begin{gathered}
P_{11}(x, \lambda) \equiv 1 . \quad \text { Similarly } P_{22}(x, \lambda) \equiv 1, \\
P_{12}(x, \lambda) \equiv 0 \equiv P_{21}(x, \lambda) .
\end{gathered}
$$

If we substitute these results in (22), we get $\varphi^{(i)}(x, \lambda) \equiv$ $\widetilde{\varphi}^{(i)}(x, \lambda), \Phi^{(i)}(x, \lambda) \equiv \widetilde{\Phi}^{(i)}(x, \lambda)(i=0,1)$ for all $x$ and $\lambda$. Therefore $L=\widetilde{L}$.

\section{The Main Equation of the Inverse Problem}

In this section, we solve the inverse problem of recovering $L$ from the given spectral data by using ideas of the contour integral method.

We denote that if $y(x, \lambda)$ and $z(x, \mu)$ are solutions of the equations $\ell y=\lambda y$ and $\ell z=\mu z$ separately, then

$$
\frac{d}{d x}\langle y, z\rangle=(\lambda-\mu) y z, \quad\langle y, z\rangle:=y z^{\prime}-y^{\prime} z .
$$

Note

$$
D(x, \lambda, \mu):=\frac{\langle\varphi(x, \lambda), \varphi(x, \mu)\rangle}{\lambda-\mu}=\int_{0}^{x} \varphi(t, \lambda) \varphi(t, \mu) d t .
$$

Let us select a model boundary value problem $\widetilde{L}=$ $L(\widetilde{q}(x), \widetilde{C})$ with real $\widetilde{q}(x) \in L_{2}(0, \pi)$ such that $C=\widetilde{C}$. Let $\left\{\tilde{\lambda}_{n}, \widetilde{\alpha}_{n}\right\}_{n \geq 0}$ be the spectral data of $\tilde{L}$.

Furthermore, let

$$
\xi_{n}:=\left|\rho_{n}-\tilde{\rho}_{n}\right|+\left|\alpha_{n}-\tilde{\alpha}_{n}\right| .
$$

It follows from (12) and (14) and analogous formulas for $\tilde{\rho}_{n}$ and $\widetilde{\alpha}_{n}$ that

$$
\Lambda:=\left(\sum_{n=0}^{\infty}\left[(n+1) \xi_{n}\right]^{2}\right)^{1 / 2}<\infty, \quad \sum_{n} \xi_{n}<\infty .
$$

Denote that

$$
\begin{gathered}
\lambda_{n 0}=\lambda_{n}, \quad \lambda_{n 1}=\tilde{\lambda}_{n}, \quad \alpha_{n 0}=\alpha_{n}, \\
\alpha_{n 1}=\widetilde{\alpha}_{n}, \quad \varphi_{n i}(x)=\varphi\left(x, \lambda_{n i}\right), \\
\widetilde{\varphi}_{n i}(x)=\widetilde{\varphi}\left(x, \lambda_{n i}\right), \quad P_{n i, j}(x)=\frac{1}{\alpha_{k j}} D\left(x, \lambda_{n i}, \lambda_{k j}\right), \\
\widetilde{P}_{n i, k j}(x)=\frac{1}{\alpha_{k j}} \widetilde{D}\left(x, \lambda_{n i}, \lambda_{k j}\right), \quad i, j \in\{0,1\}, n, k \geq 0 .
\end{gathered}
$$

Then, according to (31),

$$
\begin{aligned}
P_{n i, k j}(x) & =\frac{\left\langle\varphi_{n i}(x), \varphi_{k j}(x)\right\rangle}{\alpha_{k j}\left(\lambda_{n i}-\lambda_{k j}\right)} \\
& =\frac{1}{\alpha_{k j}} \int_{0}^{x} \varphi_{n i}(t), \varphi_{k j}(t) d t, \\
\widetilde{P}_{n i, k j}(x) & =\frac{\left\langle\widetilde{\varphi}_{n i}(x), \widetilde{\varphi}_{k j}(x)\right\rangle}{\alpha_{k j}\left(\lambda_{n i}-\lambda_{k j}\right)} \\
& =\frac{1}{\alpha_{k j}} \int_{0}^{x} \widetilde{\varphi}_{n i}(t), \widetilde{\varphi}_{k j}(t) d t .
\end{aligned}
$$

It is clear that

$$
\begin{aligned}
& P_{n i, k j}^{\prime}(x)=\frac{1}{\alpha_{k j}} \varphi_{n i}(x) \varphi_{k j}(x), \\
& \widetilde{P}_{n i, k j}^{\prime}(x)=\frac{1}{\alpha_{k j}} \widetilde{\varphi}_{n i}(x) \widetilde{\varphi}_{k j}(x) .
\end{aligned}
$$


Lemma 5 (Schwarz's lemma). Let $f(\rho)$ be an analytic function for $\left|\rho-\rho^{0}\right|<$ a such that $f\left(\rho^{0}\right)=0$ and $|f(\rho)| \leq A$. Then $|f(\rho)| \leq(A / a)\left|\rho-\rho^{0}\right|$ for $\left|\rho-\rho^{0}\right|<a$.

Lemma 6. The following estimates are valid for $x \in[0, \pi] \backslash\{d\}$, $n, k \geq 0, i, j, v=0,1$ :

$$
\begin{gathered}
\left|\varphi_{n i}(x)\right| \leq c, \quad\left|\left(\Gamma \varphi_{n i}\right)(x)\right| \leq c(n+1), \\
\left|\varphi_{n 0}(x)-\varphi_{n 1}(x)\right| \leq c \xi_{n}, \\
\left|\left(\Gamma \varphi_{n 0}\right)(x)-\left(\Gamma \varphi_{n 1}\right)(x)\right| \leq c \xi_{n}(n+1), \\
\left|P_{n i, k j}(x)\right| \leq \frac{c}{|n-k|+1}, \quad\left|P_{n i, k j}^{(v+1)}(x)\right| \leq c(n+k+1)^{v} \\
\left|P_{n i, k 0}(x)-P_{n i, k 1}(x)\right| \leq \frac{c \xi_{k}}{|n-k|+1}, \\
\left|P_{n 0, k j}(x)-P_{n 1, k j}(x)\right| \leq \frac{c \xi_{n}}{|n-k|+1}, \\
\left|P_{n 0, k 0}(x)-P_{n 1, k 0}(x)-P_{n 0, k 1}(x)+P_{n 1, k 1}(x)\right| \\
\leq \frac{c \xi_{n} \xi_{k}}{|n-k|+1} .
\end{gathered}
$$

The similar estimates are also current for $\widetilde{\varphi}_{n i}(x), \widetilde{P}_{n i, k j}(x)$.

Proof. It follows from (12), (14), (15), and (16) that $\left|\varphi_{n i}(x)\right| \leq c$, $\left|\left(\Gamma \varphi_{n i}\right)(x)\right| \leq c(n+1)$ when $x<d$ and $x>d$.

Besides, for a fixed $a>0$,

$$
\begin{gathered}
|\varphi(x, \lambda)| \leq c, \quad|(\Gamma \varphi)(x, \lambda)| \leq c(n+1), \\
\left|\rho-\rho_{n 1}\right| \leq a .
\end{gathered}
$$

Applying Schwarz's lemma in the $\rho$-plane to the function $f(\rho):=\varphi^{(v)}(x, \lambda)-\varphi^{(v)}\left(x, \lambda_{n 1}\right)$ with fixed $v, n, x$, and $a$, we obtain

$$
\begin{gathered}
\left|\varphi^{(v)}(x, \lambda)-\varphi^{(v)}\left(x, \lambda_{n 1}\right)\right| \leq c(n+1)^{v}\left|\rho-\rho_{n 1}\right|, \\
\left|\rho-\rho_{n 1}\right| \leq a .
\end{gathered}
$$

Here $(\Gamma \varphi)(x, \lambda)$ is denoted by $\varphi^{(v)}(x, \lambda)$ for $v=1$. As a result,

$$
\left|\varphi_{n 0}^{(v)}(x)-\varphi_{n 1}^{(v)}(x)\right| \leq c(n+1)^{v}\left|\rho_{n 0}-\rho_{n 1}\right|,
$$

and (37) is proved.

Let us show that

$$
\begin{aligned}
& \left|D\left(x, \lambda, \lambda_{k j}\right)\right| \leq \frac{c e^{|\tau| x}}{|\rho \mp k|+1}, \\
& \lambda=\rho^{2}, \quad \mp \operatorname{Re} \rho \geq 0, \quad \tau=\operatorname{Im} \rho, \quad k \geq 0 .
\end{aligned}
$$

For definiteness, let $\sigma:=\operatorname{Re} \rho \geq 0$. Get a fixed $\delta_{0}>0$. For $\left|\rho-\rho_{k j}\right| \geq \delta_{0}$, we have by the help of (31), (15), and (12),

$$
\left|D\left(x, \lambda, \lambda_{k j}\right)\right|=\frac{\left|\left\langle\varphi(x, \lambda), \varphi\left(x, \lambda_{k j}\right)\right\rangle\right|}{\left|\lambda-\lambda_{k j}\right|} \leq c e^{|\tau| x \mid} \frac{|\rho|+\left|\rho_{k j}\right|}{\left|\rho^{2}-\rho_{k j}^{2}\right|} .
$$

Since $\left(|\rho|+\left|\rho_{k j}\right|\right) /\left|\rho+\rho_{k j}\right| \leq\left(\sqrt{\sigma^{2}+\tau^{2}}+\left|\rho_{k j}\right|\right) /$ $\sqrt{\sigma^{2}+\tau^{2}+\rho_{k j}^{2}} \leq \sqrt{2}$, we obtain

$$
\begin{gathered}
\left|D\left(x, \lambda, \lambda_{k j}\right)\right| \leq \frac{c e^{|\tau| x}}{\left|\rho-\rho_{k j}\right|}, \\
\frac{|\rho-k|+1}{\left|\rho-\rho_{k j}\right|} \leq \frac{\left|\rho_{k j}-k\right|+1}{\left|\rho-\rho_{k j}\right|} \leq c, \\
\text { and so } \frac{1}{\left|\rho-\rho_{k j}\right|} \leq \frac{c}{|\rho-k|+1} .
\end{gathered}
$$

Thus, this yields (42) for $\left|\rho-\rho_{k j}\right| \geq \delta_{0}$. For $\left|\rho-\rho_{k j}\right| \leq \delta_{0}$,

$$
\left|D\left(x, \lambda, \lambda_{k j}\right)\right|=\left|\int_{0}^{x} \varphi(t, \lambda) \varphi\left(t, \lambda_{k j}\right) d t\right| \leq c e^{|\tau| x} .
$$

That is, (42) is also valid for $\left|\rho-\rho_{k j}\right| \leq \delta_{0}$. Likewise it can be shown that

$$
\begin{aligned}
& |D(x, \lambda, \mu)| \leq \frac{c e^{|\tau| x}}{|\rho \mp \theta|+1}, \\
& \mu=\theta^{2}, \quad|\operatorname{Im} \theta| \leq c_{0}, \quad \mp \operatorname{Re} \rho \operatorname{Re} \theta \geq 0 .
\end{aligned}
$$

Using Schwarz's lemma, we get

$$
\begin{aligned}
\left|D\left(x, \lambda, \lambda_{k 1}\right)-D\left(x, \lambda, \lambda_{k 0}\right)\right| & \leq \frac{c e^{|\tau| x} \xi_{k}}{|\rho \mp k|+1}, \\
\mp \operatorname{Re} \rho & \geq 0, \quad k \geq 0 .
\end{aligned}
$$

Particularly, this yields

$$
\left|D\left(x, \lambda_{n i}, \lambda_{k 1}\right)-D\left(x, \lambda_{n i}, \lambda_{k 0}\right)\right| \leq \frac{c \xi_{k}}{|n-k|+1} .
$$

Symmetrically,

$$
\left|D\left(x, \lambda_{n 1}, \lambda_{k j}\right)-D\left(x, \lambda_{n 0}, \lambda_{k j}\right)\right| \leq \frac{c \xi_{n}}{|n-k|+1} .
$$

If we apply Schwarz's lemma to the function

$$
Q_{k}(x, \lambda):=D\left(x, \lambda, \lambda_{k 1}\right)-D\left(x, \lambda, \lambda_{k 0}\right)
$$

for fixed $k$ and $x$, we obtain

$$
\begin{aligned}
& \mid D\left(x, \lambda_{n 0}, \lambda_{k 0}\right)-D\left(x, \lambda_{n 1}, \lambda_{k 0},\right)-D\left(x, \lambda_{n 0}, \lambda_{k 1}\right) \\
& \quad+D\left(x, \lambda_{n 1}, \lambda_{k 1}\right) \mid \leq \frac{c \xi_{n} \xi_{k}}{|n-k|+1} .
\end{aligned}
$$

These approximates together with (34), (36), (12), and (14) suggest (38). 

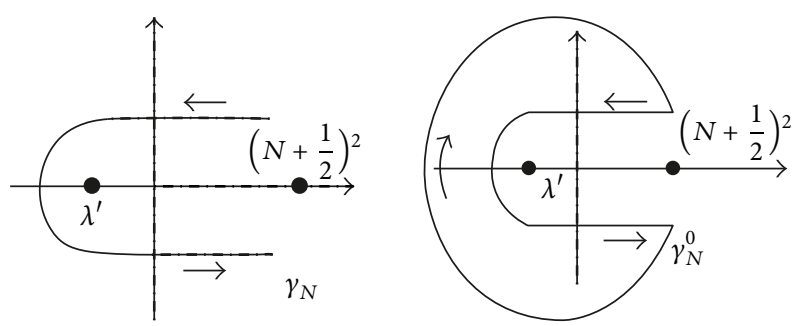

FIgURe 1

Lemma 7. The following relations hold:

$$
\begin{aligned}
& \widetilde{\varphi}(x, \lambda)=\varphi(x, \lambda) \\
& +\sum_{k=0}^{\infty}\left(\frac{\left\langle\tilde{\varphi}(x, \lambda), \tilde{\varphi}_{k 0}(x)\right\rangle}{\alpha_{k 0}\left(\lambda-\lambda_{k 0}\right)} \varphi_{k 0}(x)\right. \\
& \left.-\frac{\left\langle\widetilde{\varphi}(x, \lambda), \widetilde{\varphi}_{k 1}(x)\right\rangle}{\alpha_{k 1}\left(\lambda-\lambda_{k 1}\right)} \varphi_{k 1}(x)\right) \\
& \frac{\langle\varphi(x, \lambda), \varphi(x, \mu)\rangle}{\lambda-\mu}-\frac{\langle\widetilde{\varphi}(x, \lambda), \widetilde{\varphi}(x, \mu)\rangle}{\lambda-\mu} \\
& +\sum_{k=0}^{\infty}\left\{\frac{\left\langle\widetilde{\varphi}(x, \lambda), \widetilde{\varphi}_{k 0}(x)\right\rangle}{\alpha_{k 0}\left(\lambda-\lambda_{k 0}\right)}\right. \\
& \times \frac{\left\langle\varphi_{k 0}(x), \varphi(x, \mu)\right\rangle}{\lambda_{k 0}-\mu} \\
& -\frac{\left\langle\widetilde{\varphi}(x, \lambda), \tilde{\varphi}_{k 1}(x)\right\rangle}{\alpha_{k 1}\left(\lambda-\lambda_{k 1}\right)} \\
& \left.\times \frac{\left\langle\varphi_{k 1}(x), \varphi(x, \mu)\right\rangle}{\lambda_{k 1}-\mu}\right\}=0 .
\end{aligned}
$$

Proof. (1) Denote $\lambda^{\prime}=\min _{n i} \lambda_{n i}$ and take a fixed $\delta>0$. In the $\lambda$-plane we regard closed contours $\gamma_{N}=\gamma_{N}^{+} \cup \gamma_{N}^{-} \cup \gamma^{\prime} \cup \Gamma_{N}^{\prime}$, (with counterclockwise circuit) (see Figure 1) where

$$
\begin{gathered}
\gamma_{N}^{ \pm}=\left\{\lambda: \pm \operatorname{Im} \lambda=\delta, \operatorname{Re} \lambda \geq \lambda^{\prime},|\lambda| \leq\left(N+\frac{1}{2}\right)^{2}\right\} \\
\gamma^{\prime}=\left\{\lambda: \lambda-\lambda^{\prime}=\delta e^{i \alpha}, \alpha \in\left(\frac{\pi}{2}, \frac{3 \pi}{2}\right)\right\} \\
\Gamma_{N}^{\prime}=\Gamma_{N} \cap\{\lambda:|\operatorname{Im} \lambda| \leq \delta, \operatorname{Re} \lambda>0\}, \\
\Gamma_{N}=\left\{\lambda:|\lambda|=\left(N+\frac{1}{2}\right)^{2}\right\}
\end{gathered}
$$

Denote $\gamma_{N}^{0}=\gamma_{N}^{+} \cup \gamma_{N}^{-} \cup \gamma^{\prime} \cup\left(\Gamma_{N} \backslash \Gamma_{N}^{\prime}\right)$ (with clockwise circuit) (see Figure 1).

Let $P(x, \lambda)=\left[P_{j k}(x, \lambda)\right]_{j, k=1,2}$ be the matrix determined by (20). It follows from (21) and (17) that, for each fixed $x$, the functions $P_{j k}(x, \lambda)$ are meromorphic in $\lambda$ with simple poles $\left\{\lambda_{n}\right\}$ and $\left\{\widetilde{\lambda}_{n}\right\}$. By Cauchy's integral formula,

$$
P_{1}(x, k)-\delta_{1 k}=\frac{1}{2 \pi i} \int_{\gamma_{N}^{0}} \frac{P_{1 k}(x, \xi)-\delta_{1 k}}{\lambda-\xi} d \xi, \quad k=1,2, \ldots,
$$

where $\lambda \in \operatorname{int}\left(\gamma_{N}^{0}\right)$ and $\delta_{j k}$ is the Kronecker delta. Therefore,

$$
\begin{aligned}
P_{1}(x, k)-\delta_{1 k}= & \frac{1}{2 \pi i} \int_{\gamma_{N}} \frac{P_{1 k}(x, \xi)}{\lambda-\xi} d \xi \\
& -\frac{1}{2 \pi i} \int_{\Gamma_{N}} \frac{P_{1 k}(x, \xi)-\delta_{1 k}}{\lambda-\xi} d \xi
\end{aligned}
$$

where $\Gamma_{N}$ is used with counterclockwise circuit. Substituting into (22), we get

$$
\begin{aligned}
\varphi(x, \lambda)= & \tilde{\varphi}(x, \lambda)+\frac{1}{2 \pi i} \\
& \times \int_{\gamma_{N}} \frac{\widetilde{\varphi}(x, \lambda) P_{11}(x, \xi)+(\Gamma \widetilde{\varphi})(x, \lambda) P_{12}(x, \xi)}{\lambda-\xi} d \xi \\
& +\varepsilon_{N}(x, \lambda)
\end{aligned}
$$

where

$$
\begin{aligned}
& \varepsilon_{N}(x, \lambda) \\
& =-\frac{1}{2 \pi i} \\
& \quad \times \int_{\Gamma_{N}} \frac{\widetilde{\varphi}(x, \lambda)\left(P_{11}(x, \xi)-1\right)+(\Gamma \widetilde{\varphi})(x, \lambda) P_{12}(x, \xi)}{\lambda-\xi} d \xi .
\end{aligned}
$$

By the help of (29),

$$
\lim _{N \rightarrow \infty} \varepsilon_{N}(x, \lambda)=0
$$

uniformly with respect to $x \in[0, \pi]$ and $\lambda$ on compact sets.

If we take (25) into account, we calculate

$$
\begin{aligned}
& \varphi(x, \lambda) \\
& =\widetilde{\varphi}(x, \lambda)+\frac{1}{2 \pi i} \\
& \quad \times \int_{\gamma_{N}}\{\widetilde{\varphi}(x, \lambda)[\varphi(x, \xi)(\Gamma \widetilde{\Phi})(x, \xi)-\widetilde{\Phi}(x, \xi)(\Gamma \widetilde{\varphi})(x, \xi)] \\
& \quad+(\Gamma \widetilde{\varphi})(x, \lambda)[\Phi(x, \xi) \widetilde{\varphi}(x, \xi)-\varphi(x, \xi) \widetilde{\Phi}(x, \xi)]\} \\
& \quad \times \frac{d \xi}{\lambda-\xi}+\varepsilon_{N}(x, \lambda) .
\end{aligned}
$$


In view of (17), this supplies

$$
\begin{aligned}
\widetilde{\varphi}(x, \lambda)= & \varphi(x, \lambda)+\frac{1}{2 \pi i} \\
& \times \int_{\gamma_{N}} \frac{\langle\widetilde{\varphi}(x, \lambda), \widetilde{\varphi}(x, \xi)\rangle}{\lambda-\xi} \widehat{M}(\xi) \varphi(x, \xi) d \xi \\
& +\varepsilon_{N}(x, \lambda)
\end{aligned}
$$

where $\widehat{M}(\lambda)=M(\lambda)-\widetilde{M}(\lambda)$, since the terms with $C(x, \xi)$ vanish by Cauchy's theorem.

It follows from

$$
\operatorname{Re}_{\lambda=\lambda_{n}} M(\lambda)=-\frac{\Delta^{0}\left(\lambda_{n}\right)}{\dot{\Delta}\left(\lambda_{n}\right)}=-\frac{\beta_{n}}{\dot{\Delta}\left(\lambda_{n}\right)}=\frac{1}{\alpha_{n}}
$$

that

$$
\begin{gathered}
\operatorname{Re}_{\xi=\lambda_{k j}} \frac{\langle\tilde{\varphi}(x, \lambda), \tilde{\varphi}(x, \xi)\rangle}{\lambda-\xi} \widehat{M}(\xi) \varphi(x, \xi) \\
=\frac{\left\langle\widetilde{\varphi}(x, \lambda), \widetilde{\varphi}_{k j}(x)\right\rangle}{\alpha_{k j}\left(\lambda-\lambda_{k j}\right)} \varphi_{k j}(x) .
\end{gathered}
$$

Calculating the integral in (61) by the residue theorem and using (59) we get (52).

(2)Since $(1 /(\lambda-\mu))((1 /(\lambda-\xi))-(1 /(\mu-\xi)))=1 /((\lambda-$ $\xi)(\xi-\mu))$, we have by Cauchy's integral formula

$$
\begin{array}{r}
\frac{P_{j k}(x, \lambda)-P_{j k}(x, \mu)}{\lambda-\mu}=\frac{1}{2 \pi i} \int_{\gamma_{N}^{0}} \frac{P_{j k}(x, \xi)}{(\lambda-\xi)(\xi-\mu)} d \xi, \\
k, j=1,2, \quad \lambda, \mu \in \operatorname{int}\left(\gamma_{N}^{0}\right) .
\end{array}
$$

Considering in the same way as above and using (29), we get

$$
\begin{aligned}
\frac{P_{j k}(x, \lambda)-P_{j k}(x, \mu)}{\lambda-\mu}= & \frac{1}{2 \pi i} \int_{\gamma_{N}} \frac{P_{j k}(x, \xi)}{(\lambda-\xi)(\xi-\mu)} d \xi \\
& +\varepsilon_{N_{j k}}(x, \lambda, \mu),
\end{aligned}
$$

where $\lim _{n \rightarrow \infty} \varepsilon_{N_{j k}}(x, \lambda, \mu)=0 j, k=\overline{1, n}$. From (21) and (19), it follows that

$$
\begin{gathered}
P_{11}(x, \lambda)(\Gamma \tilde{\varphi})(x, \lambda)-P_{21}(x, \lambda) \varphi(x, \lambda)=(\Gamma \widetilde{\varphi})(x, \lambda) \\
P_{22}(x, \lambda) \varphi(x, \lambda)-P_{12}(x, \lambda)(\Gamma \varphi)(x, \lambda)=\widetilde{\varphi}(x, \lambda) \\
P(x, \lambda)\left[\begin{array}{c}
y(x) \\
y^{\prime}(x)
\end{array}\right]=\langle y(x), \widetilde{\Phi}(x, \lambda)\rangle\left[\begin{array}{c}
\varphi(x, \lambda) \\
(\Gamma \varphi)(x, \lambda)
\end{array}\right] \\
-\langle y(x), \widetilde{\varphi}(x, \lambda)\rangle\left[\begin{array}{c}
\Phi(x, \lambda) \\
(\Gamma \Phi)(x, \lambda)
\end{array}\right]
\end{gathered}
$$

for any $y(x) \in C^{1}[0,1]$.
If we take (65) and (67) into account, we calculate

$$
\begin{aligned}
& \frac{P_{j k}(x, \lambda)-P_{j k}(x, \mu)}{\lambda-\mu}\left[\begin{array}{c}
y(x) \\
y^{\prime}(x)
\end{array}\right] \\
& =\frac{1}{2 \pi i} \int_{\gamma_{N}}\left\{\langle y(x), \widetilde{\Phi}(x, \xi)\rangle\left[\begin{array}{c}
\varphi(x, \xi) \\
(\Gamma \varphi)(x, \xi)
\end{array}\right]\right. \\
& \left.-\langle y(x), \widetilde{\varphi}(x, \xi)\rangle\left[\begin{array}{c}
\Phi(x, \xi) \\
(\Gamma \Phi)(x, \xi)
\end{array}\right]\right\} \\
& \quad \times \frac{d \xi}{(\lambda-\xi)(\xi-\mu)}+\varepsilon_{N}^{0}(x, \lambda, \mu), \\
& \lim _{N \rightarrow \infty} \varepsilon_{N}^{0}(x, \lambda, \mu)=0 .
\end{aligned}
$$

According to $(20), P(x, \lambda)\left[\begin{array}{c}\widetilde{\varphi}(x, \lambda) \\ (\Gamma \tilde{\varphi})(x, \lambda)\end{array}\right]=\left[\begin{array}{c}\varphi(x, \lambda) \\ (\Gamma \varphi)(x, \lambda)\end{array}\right]$.

Thus, $\operatorname{det}\left(P(x, \lambda)\left[\begin{array}{c}\widetilde{\varphi}(x, \lambda) \\ (\Gamma \tilde{\varphi})(x, \lambda)\end{array}\right],\left[\begin{array}{c}\varphi(x, \mu) \\ (\Gamma \varphi)(x, \mu)\end{array}\right]\right)=\langle\varphi(x, \lambda), \varphi(x$, $\mu)\rangle$.

Moreover, using (66) we obtain

$$
\begin{aligned}
& \operatorname{det}\left(P(x, \mu)\left[\begin{array}{c}
\tilde{\varphi}(x, \lambda) \\
(\Gamma \widetilde{\varphi})(x, \lambda)
\end{array}\right],\left[\begin{array}{c}
\varphi(x, \mu) \\
(\Gamma \varphi)(x, \mu)
\end{array}\right]\right) \\
& \quad=\langle\widetilde{\varphi}(x, \lambda), \widetilde{\varphi}(x, \mu)\rangle .
\end{aligned}
$$

So,

$$
\begin{gathered}
\operatorname{det}\left((P(x, \lambda)-P(x, \mu))\left[\begin{array}{c}
\tilde{\varphi}(x, \lambda) \\
(\Gamma \tilde{\varphi})(x, \lambda)
\end{array}\right],\left[\begin{array}{c}
\varphi(x, \mu) \\
(\Gamma \varphi)(x, \mu)
\end{array}\right]\right) \\
=\langle\varphi(x, \lambda), \varphi(x, \mu)\rangle-\langle\widetilde{\varphi}(x, \lambda), \widetilde{\varphi}(x, \mu)\rangle .
\end{gathered}
$$

As a result, $(68)$ for $y(x)=\widetilde{\varphi}(x, \lambda)$ supplies

$$
\begin{gathered}
\frac{\langle\varphi(x, \lambda), \varphi(x, \mu)\rangle}{\lambda-\mu}-\frac{\langle\widetilde{\varphi}(x, \lambda), \widetilde{\varphi}(x, \mu)\rangle}{\lambda-\mu} \\
=\frac{1}{2 \pi i} \int_{\gamma_{N}}\left\{\frac{\langle\widetilde{\varphi}(x, \lambda), \widetilde{\Phi}(x, \xi)\rangle\langle\varphi(x, \xi), \varphi(x, \mu)\rangle}{(\lambda-\xi)(\xi-\mu)}\right. \\
\left.-\frac{\langle\widetilde{\varphi}(x, \lambda), \widetilde{\varphi}(x, \xi)\rangle\langle\Phi(x, \xi), \varphi(x, \mu)\rangle}{(\lambda-\xi)(\xi-\mu)}\right\} d \xi \\
+\varepsilon_{N}^{\prime}(x, \lambda, \mu), \lim _{N \rightarrow \infty} \varepsilon_{N}^{\prime}(x, \lambda, \mu)=0 .
\end{gathered}
$$

By virtue of (17), (62), and residue theorem, we obtain (53). Similarly, the following relation can be obtained:

$$
\begin{aligned}
& \widetilde{\Phi}(x, \lambda)=\Phi(x, \lambda) \\
&+\sum_{k=0}^{\infty}\left(\frac{\left\langle\widetilde{\varphi}(x, \lambda), \widetilde{\varphi}_{k 0}(x)\right\rangle}{\alpha_{k 0}\left(\lambda-\lambda_{k 0}\right)} \varphi_{k 0}(x)\right. \\
&\left.\quad \frac{\left\langle\widetilde{\varphi}(x, \lambda), \widetilde{\varphi}_{k 1}(x)\right\rangle}{\alpha_{k 1}\left(\lambda-\lambda_{k 1}\right)} \varphi_{k 1}(x)\right) .
\end{aligned}
$$


It follows from the definition of $\widetilde{P}_{n i, k j}(x), P_{n i, k j}(x)$ and (52), (53) that

$$
\widetilde{\varphi}_{n i}(x)=\varphi_{n i}(x)+\sum_{k=0}^{\infty}\left(\widetilde{P}_{n i, k 0}(x) \varphi_{k 0}(x)-\widetilde{P}_{n i, k 1}(x) \varphi_{k 1}(x)\right),
$$

$$
\begin{aligned}
P_{n i, l j}(x) & -\widetilde{P}_{n i, l j}(x) \\
& +\sum_{k=0}^{\infty}\left(\widetilde{P}_{n i, k 0}(x) P_{k 0, l j}(x)-\widetilde{P}_{n i, k 1}(x) P_{k 1, l j}(x)\right)
\end{aligned}
$$$$
=0 \text {. }
$$

Denote

$$
\begin{gathered}
\varepsilon_{0}(x)=\sum_{k=0}^{\infty}\left(\frac{1}{\alpha_{k 0}} \widetilde{\varphi}_{k 0}(x) \varphi_{k 0}(x)-\frac{1}{\alpha_{k 1}} \widetilde{\varphi}_{k 1}(x) \varphi_{k 1}(x)\right), \\
\varepsilon(x)=-2 \varepsilon_{0}^{\prime}(x) .
\end{gathered}
$$

Lemma 8. The series in (75) converges absolutely and uniformly on $[0, \pi]$. The function $\varepsilon_{0}(x)$ is absolutely continuous and $\varepsilon(x) \in L_{2}(0, \pi)$.

Proof. We can write $\varepsilon_{0}(x)$ in the form

$$
\varepsilon_{0}(x)=A_{1}(x)+A_{2}(x)
$$

where

$$
\begin{array}{r}
A_{1}(x)=\sum_{k=0}^{\infty}\left(\frac{1}{\alpha_{k 0}}-\frac{1}{\alpha_{k 1}}\right) \widetilde{\varphi}_{k 0}(x) \varphi_{k 0}(x), \\
A_{2}(x)=\sum_{k=0}^{\infty} \frac{1}{\alpha_{k 1}}\left\{\left(\widetilde{\varphi}_{k 0}(x)-\widetilde{\varphi}_{k 1}(x)\right) \varphi_{k 0}(x)\right. \\
\left.+\widetilde{\varphi}_{k 1}(x)\left(\varphi_{k 0}(x)-\varphi_{k 1}(x)\right)\right\} .
\end{array}
$$

It follows from (12), (14), (33), and (37) that the series in (77) converge absolutely and uniformly on $[0, \pi]$ and

$$
\left|A_{j}(x)\right| \leq c \sum_{k=0}^{\infty} \xi_{k} \leq c \Lambda, \quad j=1,2 .
$$

Moreover, using the asymptotic formulae (15), (12), (14), and (33) we calculate

$$
\begin{aligned}
A_{1}^{\prime}(x) & =\sum_{k=0}^{\infty}\left(\frac{1}{\alpha_{k 0}}-\frac{1}{\alpha_{k 1}}\right) \frac{d}{d x}\left(\widetilde{\varphi}_{k 0}(x) \varphi_{k 0}(x)\right) \\
& =\sum_{k=0}^{\infty} \gamma_{k}\left(\sin 2 k x+\frac{\eta_{k}(x)}{k+1}\right),
\end{aligned}
$$

where $\left\{\gamma_{k}\right\} \in l_{2}$ and $\max _{0 \leq x \leq \pi}\left|\eta_{k}(x)\right| \leq c$ for $k \geq 0$. Therefore, $A_{1}(x) \in W_{2}^{1}(0, \pi)$. Analogously, we obtain $A_{2}(x) \in W_{2}^{1}(0, \pi)$ and consequently $\varepsilon_{0}(x) \in W_{2}^{1}(0, \pi)$.
Lemma 9. The following relation holds:

$$
q(x)=\tilde{q}(x)+\varepsilon(x),
$$

where $\varepsilon(x)$ is defined by (75).

Proof. If we differentiate (52) twice with respect to $x$ and use (30) and (75), we obtain

$$
\begin{aligned}
& (\Gamma \tilde{\varphi})(x, \lambda)-\varepsilon_{0}(x) \tilde{\varphi}(x, \lambda) \\
& =(\Gamma \varphi)(x, \lambda)+\sum_{k=0}^{\infty}\left(\frac{\left\langle\widetilde{\varphi}(x, \lambda), \widetilde{\varphi}_{k 0}(x)\right\rangle}{\alpha_{k 0}\left(\lambda-\lambda_{k 0}\right)}\left(\Gamma \varphi_{k 0}\right)(x)\right. \\
& \left.-\frac{\left\langle\tilde{\varphi}(x, \lambda), \widetilde{\varphi}_{k 1}(x)\right\rangle}{\alpha_{k 1}\left(\lambda-\lambda_{k 1}\right)}\left(\Gamma \varphi_{k 1}\right)(x)\right), \\
& (\Gamma \tilde{\varphi})^{\prime}(x, \lambda) \\
& =(\Gamma \varphi)^{\prime}(x, \lambda) \\
& +\sum_{k=0}^{\infty}\left(\frac{\left\langle\tilde{\varphi}(x, \lambda), \tilde{\varphi}_{k 0}(x)\right\rangle}{\alpha_{k 0}\left(\lambda-\lambda_{k 0}\right)}\left(\Gamma \varphi_{k 0}\right)^{\prime}(x)\right. \\
& \left.-\frac{\left\langle\tilde{\varphi}(x, \lambda), \widetilde{\varphi}_{k 1}(x)\right\rangle}{\alpha_{k 1}\left(\lambda-\lambda_{k 1}\right)}\left(\Gamma \varphi_{k 1}\right)^{\prime}(x)\right) \\
& +2 \widetilde{\varphi}(x, \lambda) \sum_{k=0}^{\infty}\left(\frac{1}{\alpha_{k 0}} \widetilde{\varphi}_{k 0}(x)\left(\Gamma \varphi_{k 0}\right)(x)\right. \\
& \left.-\frac{1}{\alpha_{k 1}} \widetilde{\varphi}_{k 1}(x)\left(\Gamma \varphi_{k 1}\right)(x)\right) \\
& +\sum_{k=0}^{\infty}\left(\frac{1}{\alpha_{k 0}}\left[\Gamma\left(\widetilde{\varphi}(x, \lambda) \widetilde{\varphi}_{k 0}(x)\right)\right] \varphi_{k 0}(x)\right. \\
& \left.-\frac{1}{\alpha_{k 1}}\left[\Gamma\left(\widetilde{\varphi}(x, \lambda) \widetilde{\varphi}_{k 1}(x)\right)\right] \varphi_{k 1}(x)\right) \\
& +2 u(x) \tilde{\varphi}(x, \lambda) \\
& \times \sum_{k=0}^{\infty}\left(\frac{1}{\alpha_{k 0}} \tilde{\varphi}_{k 0}(x) \varphi_{k 0}(x)-\frac{1}{\alpha_{k 1}} \tilde{\varphi}_{k 1}(x) \varphi_{k 1}(x)\right) \text {. }
\end{aligned}
$$

If we replace $(\Gamma \varphi)^{\prime}(x, \lambda)$ and $(\Gamma \widetilde{\varphi})^{\prime}(x, \lambda)$ by using (1) and then replace $\varphi(x, \lambda)$ using $(52)$, then

$$
\begin{gathered}
\widetilde{q}(x) \widetilde{\varphi}(x, \lambda)=q(x) \widetilde{\varphi}(x, \lambda)+\widetilde{\varphi}(x, \lambda) 2 \varepsilon_{0}^{\prime}(x), \\
\widetilde{q}(x)=q(x)-\varepsilon(x), \\
q(x)=\widetilde{q}(x)+\varepsilon(x) .
\end{gathered}
$$

Remark 10. For each fixed $x \in[0, \pi],(73)$ can be considered as a system of linear equations with respect to $\varphi_{n i}(x) n \geq 0$, $i=0,1$. However, the series in (73) converges only "with brackets." Hence, it is not appropriate to use (73) as the main 
equation of the inverse problem. Below we will convert (73) to a linear equation in a corresponding Banach space of sequences.

Let $T$ be a set of indices $u=(n, i), n \geq 0, i=0,1$. For each fixed $x \in[0, \pi]$, we define the vector

$$
\begin{aligned}
\Omega(x) & =\left[\Omega_{u}(x)\right]_{u \in T}=\left[\begin{array}{l}
\Omega_{n 0}(x) \\
\Omega_{n 1}(x)
\end{array}\right]_{n \geq 0} \\
& =\left[\Omega_{00}, \Omega_{01}, \Omega_{10}, \Omega_{11}, \ldots\right]^{T}
\end{aligned}
$$

by the formulae

$$
\left[\begin{array}{l}
\Omega_{n 0}(x) \\
\Omega_{n 1}(x)
\end{array}\right]=\left[\begin{array}{cc}
\kappa_{n} & -\kappa_{n} \\
0 & 1
\end{array}\right]\left[\begin{array}{l}
\varphi_{n 0}(x) \\
\varphi_{n 1}(x)
\end{array}\right]
$$

$$
\begin{gathered}
=\left[\begin{array}{c}
\kappa_{n}\left(\varphi_{n 0}(x)-\varphi_{n 1}(x)\right) \\
\varphi_{n 1}(x)
\end{array}\right] \\
\kappa_{n}= \begin{cases}\xi_{n}^{-1}, & \xi_{n} \neq 0, \\
0, & \xi_{n}=0 .\end{cases}
\end{gathered}
$$

We also define the block matrix

$$
\begin{array}{r}
B(x)=\left[B_{u, v}(x)\right]_{u, v \in V}=\left[\begin{array}{ll}
B_{n 0, k 0}(x) & B_{n 0, k 1}(x) \\
B_{n 1, k 0}(x) & B_{n 1, k 1}(x)
\end{array}\right]_{n, k \geq 0}, \\
u=(n, i), \quad v=(k, j),
\end{array}
$$

by the formulae

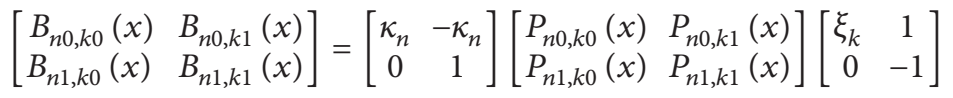

$$
\begin{aligned}
& =\left[\begin{array}{cc}
\xi_{k} \kappa_{n}\left(P_{n 0, k 0}(x)-P_{n 1, k 0}(x)\right) & \kappa_{n}\left(P_{n 0, k 0}(x)-P_{n 0, k 1}(x)-P_{n 1, k 0}(x)+P_{n 1, k 1}(x)\right) \\
\xi_{k} P_{n 1, k 0}(x) & P_{n 1, k 0}(x)-P_{n 1, k 1}(x)
\end{array}\right] .
\end{aligned}
$$

Analogously we define $\widetilde{\Omega}(x), \widetilde{B}(x)$ by replacing in the former descriptions $\varphi_{n i}(x)$ by $\widetilde{\varphi}_{n i}(x)$ and $P_{n i, k j}(x)$ by $\widetilde{P}_{n i, k j}(x)$. It follows from (37) and (38) that

$$
\begin{gathered}
\left|\Omega_{n i}^{(v)}(x)\right| \leq c(n+1)^{v}, \quad\left|B_{n i, k j}(x)\right| \leq \frac{c \xi_{k}}{|n-k|+1} \\
\left|B_{n i, k j}^{(v+1)}(x)\right| \leq c(n+k+1)^{v} \xi_{k} .
\end{gathered}
$$

Similarly,

$$
\begin{gathered}
\left|\widetilde{\Omega}_{n i}^{(v)}(x)\right| \leq c(n+1)^{v}, \quad\left|\widetilde{B}_{n i, k j}(x)\right| \leq \frac{c \xi_{k}}{|n-k|+1} \\
\left|\widetilde{B}_{n i, k j}^{(v+1)}(x)\right| \leq c(n+k+1)^{v} \xi_{k}
\end{gathered}
$$

and also

$$
\left|\widetilde{B}_{n i, k j}(x)-\widetilde{B}_{n i, k j}\left(x_{0}\right)\right| \leq c\left|x-x_{0}\right| \xi_{k}, \quad x, x_{0} \in[0, \pi],
$$

where $c$ does not depend on $x, x_{0}, n, i, j$, and $k$.

Let us consider the Banach space $m$ of bounded sequences $\alpha=\left[\alpha_{u}\right]_{u \in T}$ with the norm $\|\alpha\|_{m}=\sup _{u \in T}\left|\alpha_{u}\right|$. It follows from (87) and (88) that, for each fixed $x \in[0, \pi]$, the operators $E+\widetilde{B}(x)$ and $E-B(x)$, such that $E$ is the identity operator mapping from $m$ to $m$, are linear bounded operators and

$$
\|B(x)\|,\|\widetilde{B}(x)\| \leq c \sup _{n} \sum_{k} \frac{\xi_{k}}{|n-k|+1}<\infty .
$$

Theorem 11. In the Banach space $m$, the vector $\Omega(x) \in m$ satisfies the equation

$$
\widetilde{\Omega}(x)=(E+\widetilde{B}(x)) \Omega(x)
$$

for each fixed $x \in[0, \pi]$. Furthermore, the operator $E+\widetilde{B}(x)$ has a bounded inverse operator; that is, (91) is uniquely solvable.

Proof. We can write (73) in the form

$$
\begin{aligned}
& \widetilde{\varphi}_{n 0}(x)-\widetilde{\varphi}_{n 1}(x)=\varphi_{n 0}(x)-\varphi_{n 1}(x) \\
&+\sum_{k=0}^{\infty}\{\left(\widetilde{P}_{n 0, k 0}(x)-\widetilde{P}_{n 1, k 0}(x)\right) \\
& \times\left(\varphi_{k 0}(x)-\varphi_{k 1}(x)\right) \\
&+\left(\widetilde{P}_{n 0, k 0}(x)-\widetilde{P}_{n 1, k 0}(x)\right. \\
&\left.-\widetilde{P}_{n 0, k 1}(x)+\widetilde{P}_{n 1, k 1}(x)\right) \\
&\left.\times \varphi_{k 1}(x)\right\}, \\
& \widetilde{\varphi}_{n 1}(x)=\varphi_{n 1}(x) \\
&+\sum_{k=0}^{\infty}\left\{\widetilde{P}_{n 1, k 0}(x)\left(\varphi_{k 0}(x)-\varphi_{k 1}(x)\right)\right. \\
&\left.+\left(\widetilde{P}_{n 1, k 0}(x)-\widetilde{P}_{n 1, k 1}(x)\right) \varphi_{k 1}(x)\right\} .
\end{aligned}
$$

Considering our notations, we get

$$
\widetilde{\Omega}_{n i}(x)=\Omega_{n i}(x)+\sum_{k, j} \widetilde{B}_{n i, k j}(x) \Omega_{k j}(x), \quad(n, i),(k, j) \in T
$$


which is equivalent to (91). The series in (94) converges absolutely and uniformly for $x \in[0, \pi]$. Analogously, (74) takes the form

$$
\begin{array}{r}
B_{n i, k j}(x)-\widetilde{B}_{n i, k j}(x)+\sum_{l, s} \widetilde{B}_{n i, l s}(x) B_{l s, k j}(x)=0, \\
(n, i),(k, j),(l, s) \in T
\end{array}
$$

or

$$
(E+\widetilde{B}(x))(E-B(x))=E .
$$

Replacing $L$ and $\widetilde{L}$, similarly, we get

$$
\begin{gathered}
\Omega(x)=(E-B(x)) \widetilde{\Omega}(x), \\
(E-B(x))(E+\widetilde{B}(x))=E .
\end{gathered}
$$

Therefore, the operator $(E+\widetilde{B}(x))^{-1}$ exists and it is a linear bounded operator.

Equation (91) is called the main equation of the inverse problem. Solving (91), we find the vector $\Omega(x)$ and as a result the functions $\varphi_{n i}(x)$. we can construct the function $q(x)$ due to $\varphi_{n i}(x)=\varphi\left(x, \lambda_{n i}\right)$ are the solutions of (1). So, we get the following algorithm for the solution of inverse problem.

Algorithm 12. Given the numbers $\left\{\lambda_{n}, \alpha_{n}\right\}_{n \geq 0}$. First, select $\widetilde{L}$ such that $C=\widetilde{C}$ and construct $\widetilde{\Omega}(x), \widetilde{B}(x)$. Second, find $\Omega(x)$ by solving (91). Finally, calculate $q(x)$ by (75) and (80).

\section{Conflict of Interests}

The authors declare that there is no conflict of interests regarding the publication of this paper.

\section{References}

[1] V. Ambarzumian, "Über eine Frage der Eigenwerttheorie," Zeitschrift für Physik, vol. 53, no. 9-10, pp. 690-695, 1929.

[2] G. Borg, "Eine Umkehrung der Sturm-Liouvilleschen Eigenwertaufgabe-Bestimmung der Differentialgleichung durch die Eigenwerte," Acta Mathematica, vol. 78, no. 1, pp. 1-96, 1946.

[3] N. Levinson, “The inverse Sturm-Liouville problem," Matematisk Tidsskrift B, vol. 25, pp. 25-30, 1949.

[4] B. M. Levitan, Generalized Translation Operators and Some of Its Applications, I.P.S.T., Jerusalem, 1964.

[5] I. M. Gelfand and B. M. Levitan, "On determination of a differential equation by its spectral function," Izvestiya Akademii Nauk SSSR, vol. 15, pp. 309-360, 1951 (Russian).

[6] M. G. Gasimov and B. M. Levitan, "About Sturm-Liouville differential operators," Matematicheskii Sborn, vol. 63, no. 3, p. 105, 1964.

[7] A. N. Tikhonov, "Uniqueness theorems for jeophysics problems," Doklady Akademii Nauk SSSR, vol. 69, no. 4, pp. 797-800, 1949.

[8] R. O. Hryniv and Y. V. Mykytyuk, "Inverse spectral problems for Sturm-Liouville operators with singular potentials," Inverse Problems, vol. 19, no. 3, pp. 665-684, 2003.
[9] A. M. Savchuk and A. A. Shkalikov, "Sturm-Liouville operators with singular potentials," Matematicheskie Zametki, vol. 66, pp. 891-912, 1999 (Russian).

[10] V. A. Marchenko, "Some problems in the theory of secondorder differential operators," Doklady Akademii Nauk SSSR, vol. 72, pp. 457-560, 1950.

[11] S. Albeverio and P. Kurasov, Singular Perturbations of Differential Operators, Solvable Schrödinger Type Operators, Cambridge University Press, Cambridge, UK, 2000.

[12] J. Pöschel and E. Trubowitz, Inverse Spectral Theory, vol. 130 of Pure and Applied Mathematics, Academic Press, Orlando, Fla, USA, 1987.

[13] N. Topsakal, "Inverse problems for Sturm-Liouville operator with Coulomb potential inside an interval, Ph.D. Thesis, Cumhuriyet University," 2008. 


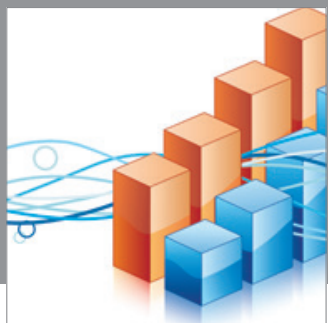

Advances in

Operations Research

mansans

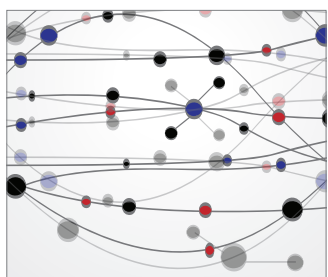

The Scientific World Journal
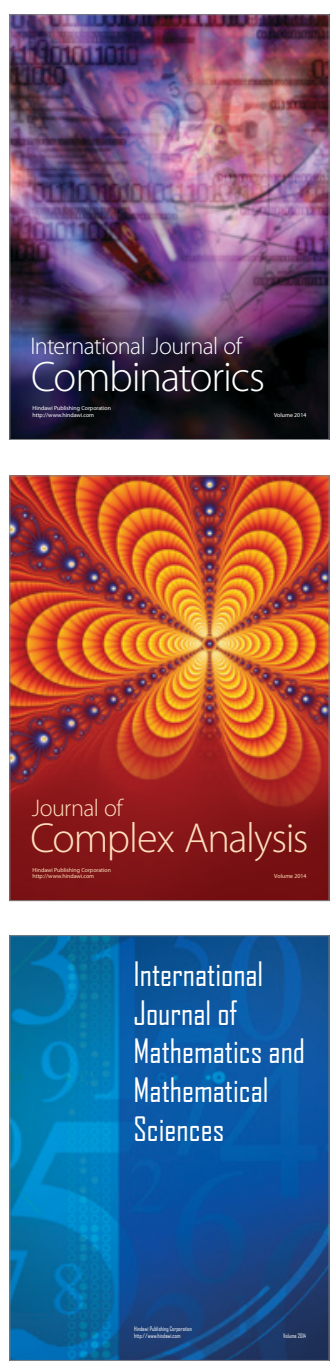
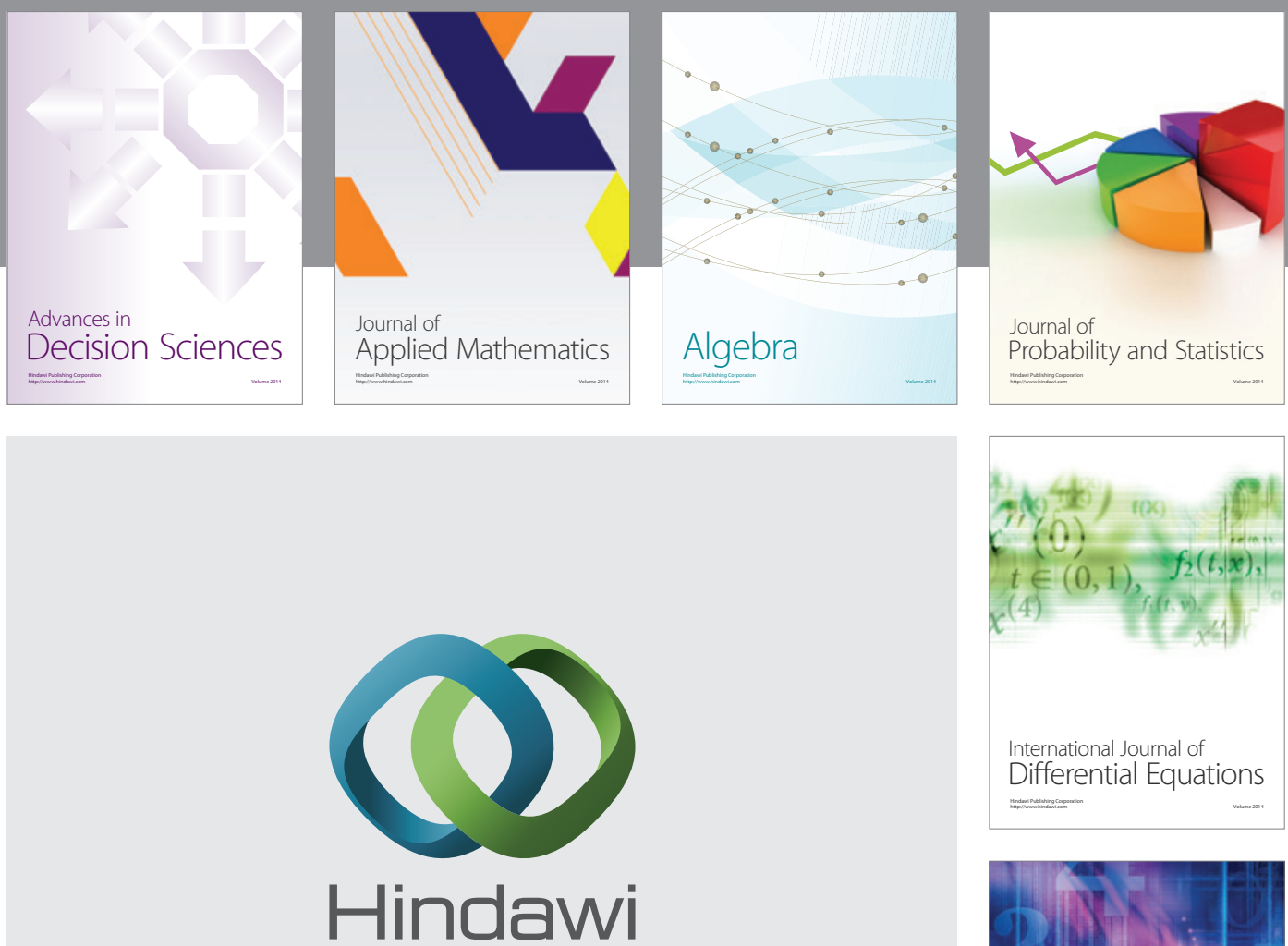

Submit your manuscripts at http://www.hindawi.com
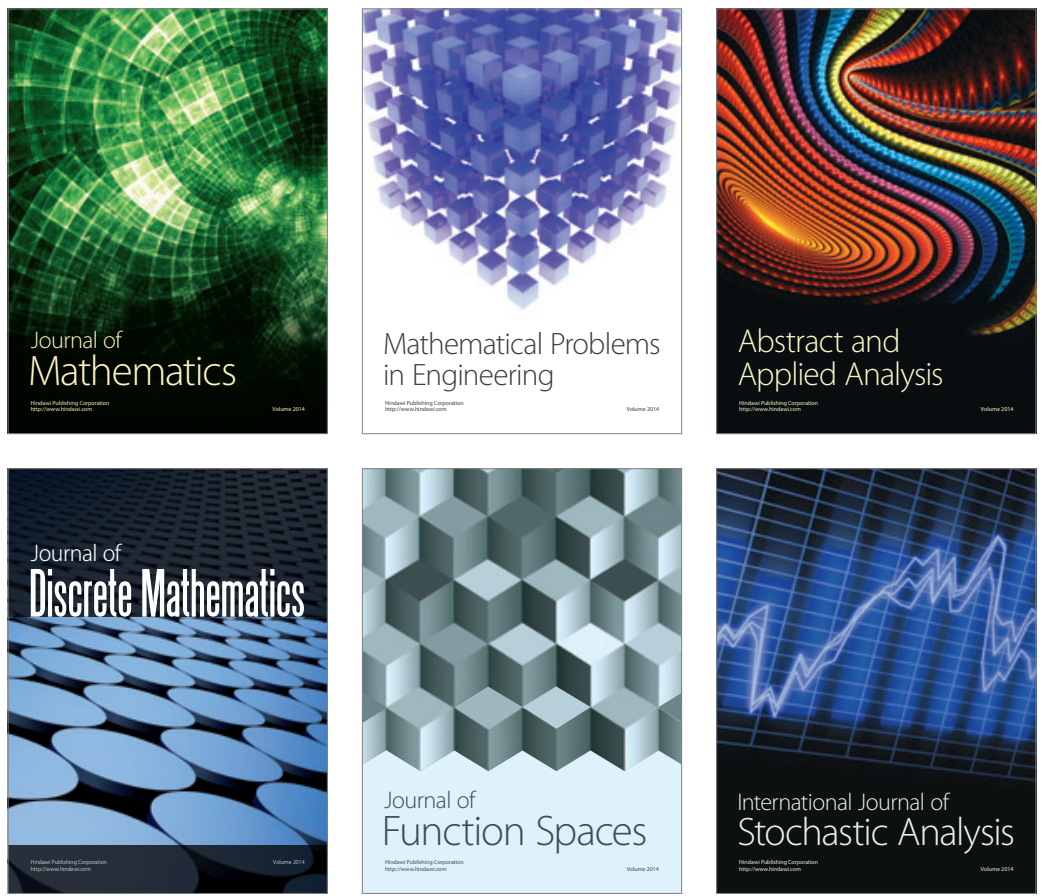

Journal of

Function Spaces

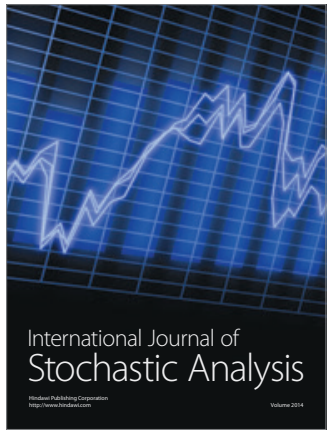

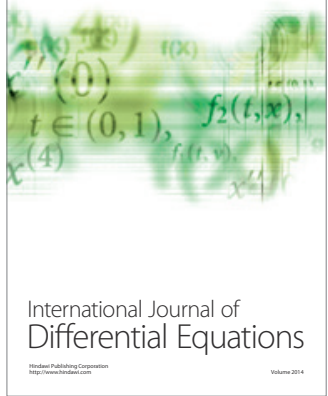
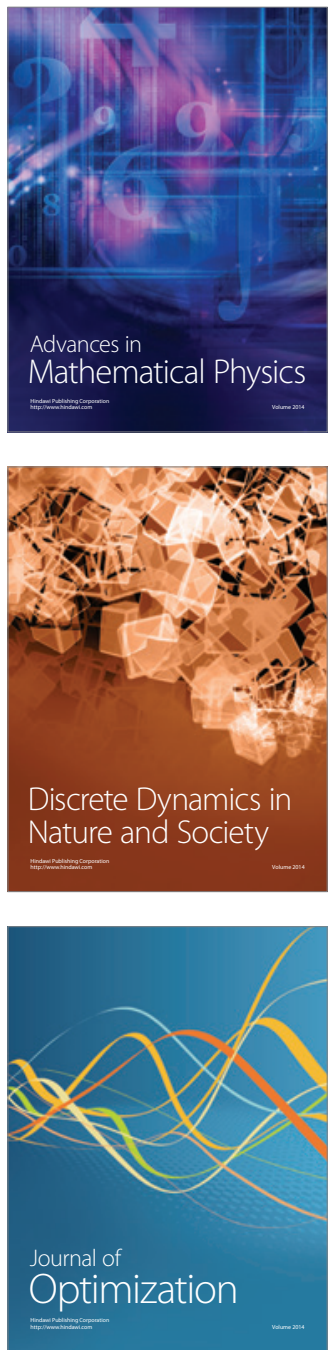